\title{
Kann zu viel Kundenorientierung nachteilig sein? Eine Analyse der Wirkung der Kundenorientierung von Verkäufern auf die Kaufentscheidung
}

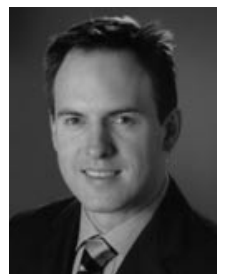

\section{Alexander Haas*}

\section{Überblick}

- Gemeinhin wird angenommen, dass die Kundenorientierung von Verkäufern deren Verkaufserfolg erhöht. Diesen Zusammenhang kann die Wissenschaft bisher nicht bestätigen.

- Der Beitrag betrachtet den Einfluss des kundenorientierten Verkäuferverhaltens auf die in einer Verkaufsinteraktion anstehende Kaufentscheidung der Kunden. Die Betrachtung erfolgt auf der Ebene der Dimensionen des kundenorientierten Verkäuferverhaltens. Deren direkte Wirkungen auf die Kaufentscheidung und deren indirekte Wirkungen auf die Kaufentscheidung über die Kundenzufriedenheit werden analysiert.

- Für die Wissenschaft zeigen die Ergebnisse, dass der Zusammenhang zwischen kundenorientiertem Verkäuferverhalten und der Kaufentscheidung der Kunden durch nach Art und Richtung unterschiedliche Teileffekte geprägt ist. Der Praxis machen die Ergebnisse zum Wirkmechanismus der Kundenorientierung auf die Kaufentscheidung deutlich, dass kundenorientierte Verkäufer ihren Verkaufserfolg durch einen gewissen Abschlussdruck verbessern können.

Keywords Customer orientation $\cdot$ personal selling $\cdot$ salespeople $\cdot$ selling effectiveness

Eingegangen: 8. März 2007

JEL: M31

Prof. Dr. Alexander Haas ( $₫)$

ist Assistenzprofessor für Marketing am Institut für Marketing und Unternehmensführung der Universität Bern. Seine Forschungsschwerpunkte sind Dienstleistungsmarketing, Kundenorientierung sowie Persönlicher Verkauf und Verkaufsmanagement. Institut für Marketing und Unternehmensführung, Abteilung Marketing, Universität Bern, Engehaldenstrasse 4, CH - 3012 Bern, Tel.: +41-(0)31-631 4541; Fax: +41-(0)31-631 8032; E-mail: Haas@imu.unibe.ch, URL: www.marketing.imu.unibe.ch. 


\section{Einleitung}

Da die Praxis die Umsetzung kundenorientierter Strategien am Markt als zentralen Erfolgsfaktor ansieht, bemühen sich Unternehmen intensiv um die Kundenorientierung ihres Verkaufspersonals (vgl. z.B. Albers 1989; Bruhn 2003, S. 1; Hennig-Thurau 2004; Homburg 1998; Stock/Hoyer 2005; Stock-Homburg 2008). Diesem Bemühen liegt die Annahme zugrunde, dass die Kundenorientierung der Verkäufer deren Verkaufserfolg positiv beeinflusst. Kundenorientiertes Verkäuferverhalten meint dabei solches Verhalten, das auf langfristige Kundenzufriedenheit zielt (vgl. Donavan/Brown/Mowen 2004; Saxe/ Weitz 1982). Danach legen sehr kundenorientierte Verkäufer in ihren Verkaufsgesprächen drei Verhaltensweisen an den Tag: Sie unterstützen die Kunden beim Lösen ihres Kaufproblems (z.B. durch akkurate Erläuterung der Produkte), bauen ein positives Gesprächsklima auf (z.B. durch zuvorkommendes Verhalten) und vermeiden den Aufbau von Abschlussdruck (z.B. indem sie auf Abschlusstechniken verzichten) (Haas 2006a).

Bisher kann die Wissenschaft die Annahme eines positiven Einflusses des kundenorientierten Verkaufens auf den Verkaufserfolg jedoch nicht bestätigen (vgl. Franke/Park 2006, S. 698). Wie die Bestandsaufnahme noch zeigen wird, liegt dies insbesondere daran, dass die bisherigen Studien unterstellen, dass die Kundenorientierung eines Verkäufers einen positiven Einfluss auf zukünftige Kaufentscheidungen des Kunden und keine negativen Effekte auf die in der gegenwärtigen Verkaufsinteraktion anstehende Kaufentscheidung des Kunden ausübt.

Wie in der Bestandsaufnahme im Detail ausgeführt, sind beide Annahmen problematisch: Die erste Annahme lässt außer Acht, dass eine Habitualisierung des Kaufverhaltens den Einfluss der verkäuferseitigen Kundenorientierung auf zukünftige Kaufentscheidungen aufheben kann (vgl. Bunn 1993; Kroeber-Riel/Weinberg 2003, S. 401ff.). Insofern ergeben sich die langfristigen Einflüsse der Kundenorientierung von Verkäufern auf den Verkaufserfolg insbesondere durch die Wirkung des kundenorientierten Verhaltens auf zukünftige nicht habitualisierte Kaufentscheidungen der Kunden. Damit kann man die langfristige Wirkung der Kundenorientierung auf den Verkaufserfolg der Verkäufer im Wesentlichen als Summe der Wirkungen des kundenorientierten Verhaltens auf die in diesen Verkaufsinteraktionen jeweils dann anstehende (nicht habitualisierte) Kaufentscheidung auffassen. Damit entscheidet gleichzeitig die Richtigkeit der zweiten Annahme, also dass die Kundenorientierung der Verkäufer keine negativen Effekte auf die in einer Verkaufsinteraktion anstehende Kaufentscheidung der Kunden ausübt, maßgeblich darüber, ob eine hohe Kundenorientierung von Verkäufern einen positiven Effekt auf den langfristigen Verkaufserfolg ausübt.

Auch die zweite Annahme ist problematisch. Denn sie berücksichtigt nicht, dass konzeptionelle Überlegungen in der Literatur auf negative Teileffekte einzelner Dimensionen des kundenorientierten Verkäuferverhaltens auf die Kaufentscheidung der Kunden hinweisen (vgl. Brown 1990, S. 18f.; Hawes/Strong/Winick 1996, S. 350). Dort wird insbesondere angemerkt, dass die Kaufwahrscheinlichkeit von Kunden höher ist, wenn die Verkäufer Abschlussdruck nicht vollständig vermeiden, sondern in gewissem Umfang aufbauen. Dies deckt sich mit aktuell in der Praxis diskutierten Erfahrungen, nach denen eine reine Kundenorientierung der Verkäufer ihrer Verkaufsaufgabe abträglich ist und daher von kundenorientierten Verkäufern ein stärkeres Anstreben von Verkaufsabschlüs- 
sen gefordert wird (vgl. Limbeck 2005). Ob negative Teileffekte der Kundenorientierung von Verkäufern auf die Kaufentscheidung der Kunden existieren, hat die Wissenschaft bisher nicht untersucht.

Vor diesem Hintergrund besteht das Ziel dieses Beitrags darin, den Einfluss des kundenorientierten Verkaufens auf anstehende Kaufentscheidungen der Kunden zu untersuchen. Dabei erfolgt die Untersuchung auf der Ebene der Dimensionen des kundenorientierten Verkaufens und unter Berücksichtigung sowohl direkter Effekte als auch indirekter Effekte über die Kundenzufriedenheit. Dadurch wird es möglich, mögliche negative Teileffekte des kundenorientierten Verkäuferverhaltens auf die Kaufentscheidung der Kunden zu identifizieren.

\section{Bestandsaufnahme}

Die Forschung hat sich intensiv mit der Kundenorientierung von Mitarbeitern beschäftigt (vgl. Haas 2008; Franke/Park 2006; Schwepker 2003). Die Arbeiten lassen sich im Wesentlichen der Dienstleistungsforschung (vgl. z.B. Brown et al. 2002; Donavan/Brown/ Mowen 2004; Hennig-Thurau 2004) und der Verkaufsforschung (vgl. z.B. Siguaw/ Brown/Widing 1994; Stock 2002; Stock/Hoyer 2002) zuordnen. Dabei basiert ein Großteil der Arbeiten auf dem von Saxe/Weitz (1982) eingeführten Konstrukt des kundenorientierten Verkaufens. Dieses wird verstanden als ,the degree to which salespeople ... [try] to help their customers make purchase decisions that will satisfy customer needs" (Saxe/Weitz 1982, S. 344).

Die Forschungsbemühungen haben zu zahlreichen Erkenntnissen über die Wirkung des kundenorientierten Verkäuferverhaltens auf die Kaufentscheidung der Kunden geführt (vgl. z.B. Baldauf/Cravens 1999; Flaherty 1999; George 1991; Howe/Hoffman/ Hardigree 1994; Keillor/Parker/Pettijohn 2000). Diese Erkenntnisse sind allerdings nicht eindeutig. Als Folge kann eine jüngste Meta-Analyse diesen Zusammenhang nicht als signifikant ausweisen (vgl. Franke/Park 2006, S. 698).

Angesichts des in der Literatur gemeinhin unterstellten positiven Zusammenhangs zwischen Kundenorientierung der Verkäufer und ihrem Verkaufserfolg (vgl. Schwepker 2003, S. 151) deutet der Befund des nicht signifikanten Zusammenhangs darauf hin, dass in den bisherigen Studien die positiven Effekte des kundenorientierten Verkaufens auf die Kaufentscheidung der Kunden geringer sind, als in der Literatur angenommen, und/oder die negativen Effekte des kundenorientierten Verkaufens auf die Kaufentscheidung der Kunden größer sind, als in der Literatur angenommen. Die Effekte können sich dabei auf die in einer konkreten Verkaufsinteraktion anstehende Kaufentscheidung, die Wahrscheinlichkeit zukünftiger Kaufentscheidungen und den Umfang der Kaufentscheidungen beziehen (vgl. Saxe/Weitz 1982).

Hinsichtlich der Wirkung der Kundenorientierung von Verkäufern auf die in einer konkreten Verkaufsinteraktion anstehende Kaufentscheidung vernachlässigt die Kundenorientierungsforschung mögliche negative Teileffekte des kundenorientierten Verkaufens auf die Kaufentscheidung der Kunden (vgl. z.B. Niebisch 1993; Saxe/Weitz 1982). Dies steht im Widerspruch zu anderen Teilen der Literatur, in der sich mit Blick auf die einzelnen Dimensionen des kundenorientierten Verkäuferverhaltens abweichende Erkenntnisse 
finden. So wird für die Dimension der Vermeidung von Abschlussdruck argumentiert, dass ein gewisser Abschlussdruck zum Auslösen der Kaufentscheidung nötig sein kann (vgl. Brown 1990, S. 18f.; Hawes/Strong/Winick 1996, S. 350). Entsprechend wäre davon auszugehen, dass zwischen Vermeidung von Abschlussdruck und Kaufentscheidung der Kunden nicht nur ein positiver, sondern auch ein negativer Zusammenhang besteht. Diese gegenläufigen Teileffekte können die Ursache dafür sein, dass Boles et al. (2001, S. 7) einen nicht signifikanten Zusammenhang zwischen Verkaufsorientierung des Verkäufers und seiner Effektivität finden.

Auch für die Konstruktdimension des Aufbaus eines positiven Gesprächsklimas wird in der Literatur für gegenläufige Effekte auf die Kaufentscheidung der Kunden argumentiert (vgl. Brown 1990, S. 18). Danach kann sich neben dem positiven Effekt ein negativer Effekt dadurch ergeben, dass die Kunden dieses Bemühen als reines Mittel des Verkäufers zum Erreichen seines Verkaufsziels ansehen und als Folge von einem Kauf absehen. Empirische Studien weisen den Zusammenhang zwischen dem Aufbau eines positiven Gesprächsklimas und der anstehenden Kaufentscheidung von Kunden sowohl mit positivem als auch mit negativem Vorzeichen aus (vgl. Evans et al. 2000, S. 522; Niebisch 1993, S. 320). Als Konsequenz ist dieser Zusammenhang bisher unklar.

Auf die Wahrscheinlichkeit zukünftiger Kaufentscheidungen unterstellt die Literatur einen positiven Einfluss der Kundenorientierung von Verkäufern (vgl. Saxe/Weitz 1982, S. 348). Diese Unterstellung vernachlässigt allerdings Erkenntnisse der konsumentenbezogenen und organisationalen Kaufverhaltensforschung, die auf Faktoren hinweisen, die diesen Einfluss negativ beeinflussen und im Ergebnis auflösen können: Zum einen sind sowohl bei Konsumenten als auch bei organisationalen Käufern zahlreiche situative Einflüsse (z.B. ein ,plötzliches“ preisgünstigeres Konkurrenzangebot) denkbar, die selbst im Falle der kundenseitigen Absicht eines zukünftigen (Wieder-)Kaufs den tatsächlichen (Wieder-)Kauf verhindern können (vgl. Ajzen/Fishbein 1977; Sheth 1973). Zum anderen kann nach einer Kaufentscheidung kundenseitig eine Habitualisierung des zukünftigen Kaufverhaltens einsetzen (z.B. wenn Konsumenten die abgeschlossene Versicherung jährlich ,automatisch“ verlängern oder es sich bei Unternehmen nach der Kaufentscheidung zukünftig um Routineeinkäufe handelt; vgl. Bunn 1993; Kroeber-Riel/Weinberg 2003, S. 401ff.) und dafür sorgen, dass sich der Einfluss des Verkäuferverhaltens auf die Kaufentscheidung der Kunden - selbst wenn er anfangs gegeben ist - langfristig auflöst.

Der aus dieser Argumentation folgende abnehmende Einfluss der Kundenorientierung von Verkäufern auf die Kaufentscheidung lässt sich risikotheoretisch erklären (vgl. Bauer 1967). Aus dieser Perspektive nehmen Kunden vor einer Kaufentscheidung eine Beratung in Anspruch, weil sie das Bedürfnis nach Risikoreduktion verspüren (vgl. z.B. Olshavsky 1973; Roselius 1971). Entsprechend ergibt sich der Einfluss der Kundenorientierung auf die Kaufentscheidung dadurch, dass das kundenseitig vorhandene Risiko durch die Kundenorientierung der Verkäufer hinreichend reduziert wird und so der Kaufentscheidung nicht mehr im Wege steht. Während das kundenseitige Risiko am Anfang der Kundenbeziehung aufgrund fehlender Informationen (z.B. über das Produkt, den Verkäufer, das Unternehmen) hoch ist, nimmt dieses Risiko durch den Aufbau von Erfahrung im Laufe der Kundenbeziehung tendenziell ab (vgl. z.B. Crosby/Evans/Cowles 1990). Somit ergibt sich aus dieser theoretischen Perspektive ein im Zeitverlauf tendenziell sinkender Einfluss der Kundenorientierung auf die Kaufentscheidung. Zudem folgt daraus, dass in zukünf- 
tigen Verkaufsinteraktionen nur dann ein Einfluss der Kundenorientierung auf die Kaufentscheidung besteht, wenn die Kunden bezüglich ihrer Entscheidung ein hinreichend großes Risiko verspüren und dieses durch eine Interaktion mit dem Verkäufer reduzieren wollen und insofern (noch) keine Habitualisierung ihres Kaufverhaltens erfolgt ist.

Als Folge ergeben sich die langfristigen Einflüsse der Kundenorientierung von Verkäufern auf ihren Verkaufserfolg insbesondere durch die zukünftigen nicht habitualisierten Kaufentscheidungen der Kunden, nämlich durch die Wirkung des kundenorientierten Verkäuferverhaltens auf diese Kaufentscheidungen in den entsprechenden Verkaufsinteraktionen. Folglich ist für die langfristige Wirkung der Kundenorientierung auf den Verkaufserfolg insbesondere die Wirkung des kundenorientierten Verkäuferverhaltens auf nicht habitualisierte, in den konkreten Verkaufsinteraktionen anstehende Kaufentscheidungen der Kunden maßgeblich.

Mit Blick auf den Umfang der Kaufentscheidungen unterstellt die Literatur gegenläufige Effekte, die den Gesamteffekt reduzieren und aufheben können (vgl. z.B. Saxe/Weitz 1982, S. 348). Denn den positiven Effekten durch das Anbieten umfassenderer Problemlösungen im Zuge der besseren Kundenkenntnis stehen negative gegenüber durch den höheren Zeiteinsatz, um die bessere Kundenkenntnis zu erreichen (z.B. durch eine intensivere Vorbereitung auf die Verkaufsinteraktion oder durch ein intensiveres Identifizieren der Kundenbedürfnisse in der Verkaufsinteraktion). Weniger kundenorientierte Verkäufer können diese Zeit für zusätzliche Verkaufsinteraktionen nutzen und insofern einen möglichen Umsatznachteil pro Kunde durch mehr Verkaufsabschlüsse kompensieren. Diese gegenläufigen Effekte wurden in den bisherigen Studien vernachlässigt und in der Literatur bisher nicht differenziert untersucht.

Wie die Bestandsaufnahme gezeigt hat, kommt dem Zusammenhang zwischen kundenorientiertem Verkaufen und (nicht habitualisierter) anstehender Kaufentscheidung der Kunden eine hohe Bedeutung für die langfristige Wirkung der Kundenorientierung von Verkäufern auf deren Verkaufsergebnis zu. Dennoch hat die Wissenschaft bisher nicht differenziert untersucht, wie sich dieser Zusammenhang darstellt. Auf Basis der Erkenntnisse der Bestandsaufnahme wird dieser Frage im Anschluss nachgegangen. Dabei wird es als geeignete Vorgehensweise angesehen, die Wirkung des kundenorientierten Verkaufens auf der Ebene der Dimensionen des Konstrukts und unter Berücksichtigung möglicher gegenläufiger Effekte zu betrachten.

\section{Entwurf eines theoretischen Bezugsrahmens}

\subsection{Der theoretische Bezugsrahmen im Überblick}

Der vorliegende Beitrag untersucht den Einfluss des kundenorientierten Verkaufens auf die in einer Verkaufsinteraktion anstehende Kaufentscheidung der Kunden. Das Konstrukt des kundenorientierten Verkaufens wird in Übereinstimmung mit jüngeren Erkenntnissen zu den Facetten des kundenorientierten Verkäuferverhaltens durch drei Dimensionen konzeptualisiert: durch Entscheidungsunterstützung, Aufbau eines positiven Gesprächsklimas und Vermeidung von Abschlussdruck (vgl. Dixon 1999; Donavan/Brown/Mowen 2004; Haas 2008; Saxe/Weitz 1982). 
Die Entscheidungsunterstützung wird definiert als das Ausmaß des verkäuferseitigen Bemühens, den Kunden beim Lösen seines Kaufproblems zu unterstützen (vgl. Reid/ Pullins/Plank 2002, S. 209; Saxe/Weitz 1982, S. 344). Diese Unterstützung kann sowohl indirekt erfolgen - durch das Gewinnen relevanter Informationen - als auch direkt - durch das Vermitteln produkt- und auswahlbezogener Informationen. Der Aufbau eines positiven Gesprächsklimas meint das Ausmaß des verkäuferseitigen Bemühens, das Gesprächsklima zwischen Verkäufer und Kunde für den Kunden möglichst angenehm zu gestalten (vgl. Donavan/Brown/Mowen 2004, S. 132; Lee/Dubinsky 2003, S. 24; Niebisch 1993, S. 248). Dieses Verhalten manifestiert sich speziell darin, als Verkäufer angenehm aufzutreten, eine persönliche Beziehung zum Kunden aufzubauen sowie dessen Wertschätzung zum Ausdruck zu bringen. Die Vermeidung von Abschlussdruck wird definiert als das Ausmaß des verkäuferseitigen Bemühens, eine Beeinflussung des Kunden zum Herbeiführen einer unmittelbaren Kaufentscheidung zu vermeiden (vgl. Niebisch 1993, S. 250; Saxe/Weitz 1982, S. 344). Es bezieht sich somit sowohl auf entsprechende Überzeugungs- als auch auf Überredungsversuche des Verkäufers.

Wie aus Abbildung 1 ersichtlich ist, wird unterstellt, dass die Wirkung der drei Dimensionen des kundenorientierten Verkaufens auf die anstehende Kaufentscheidung der Kunden über die Kundenzufriedenheit mit der Beratung (Beratungszufriedenheit) als Mediator erfolgt. Zudem wird ein direkter Effekt der Vermeidung von Abschlussdruck auf die Kaufentscheidung der Kunden unterstellt. ${ }^{1}$

Die Betrachtung sowohl einer indirekten Wirkung als auch einer direkten Wirkung der Vermeidung von Abschlussdruck auf die kurzfristige Kaufentscheidung ermöglicht die

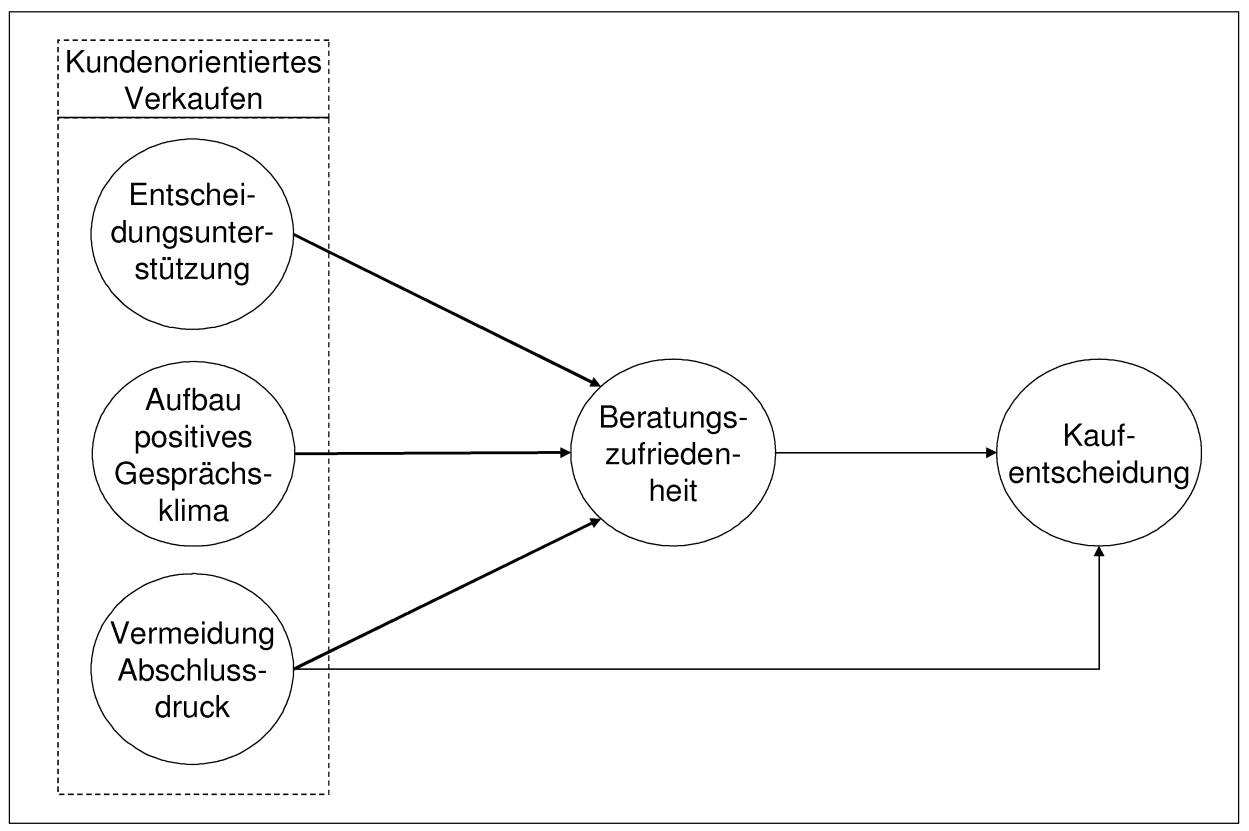

Abb. 1. Der theoretische Bezugsrahmen im Überblick 
Berücksichtigung möglicher gegenläufiger Effekte. Der indirekte Einfluss des kundenorientierten Verkaufens auf die kurzfristige Kaufentscheidung über die Beratungszufriedenheit wird zudem durch zahlreiche Befunde gestützt, die einen Einfluss des kundenorientierten Verkaufens auf die Kundenzufriedenheit einerseits und der Kundenzufriedenheit auf Kaufabsicht und -verhalten andererseits nachweisen (vgl. z.B. Goff et al. 1997, S. 178; Homburg/Bucerius 2001, S. 55f.; Stock/Hoyer 2005, S. 539; Verhoef/ Franses/Donkers 2002, S. 130).

\subsection{Fundierung der Hypothesen zu den mediierten Effekten}

Die ersten Hypothesen betreffen die Wirkungen der drei Dimensionen des kundenorientierten Verkaufens auf die Beratungszufriedenheit. Es wird unterstellt, dass Entscheidungsunterstützung, Aufbau eines positiven Gesprächsklimas und Vermeidung von Abschlussdruck einen positiven Einfluss auf die Beratungszufriedenheit ausüben.

Die Begründung der Effekte erfolgt auf Basis des Confirmation/Disconfirmation (C/D)Paradigmas (vgl. Churchill/Suprenant 1982; Homburg/Stock 2001, S. 20ff.). Das C/DParadigma unterstellt, dass die Kunden die erwartete Leistung (Soll-Leistung) und die wahrgenommene Leistung (Ist-Leistung) vergleichen. Die Erfüllung der Kundenerwartungen durch die Ist-Leistung führt zu Kundenzufriedenheit. Anderenfalls stellt sich Unzufriedenheit ein.

Vor diesem Erklärungshintergrund wird davon ausgegangen, dass Kunden die wahrgenommenen Verhaltensweisen der Verkäufer (Ist-Leistung) mit ihrer Erwartung an das Verkäuferverhalten in einer Beratungssituation (Soll-Leistung) vergleichen. Erfüllt der Verkäufer mit seinem Verhalten die Kundenerwartungen, stellt sich beim Kunden Zufriedenheit mit der Beratung ein, während die Nichterfüllung der entsprechenden Erwartungen bei diesem zu Unzufriedenheit führt. Da Kunden in Interaktionen mit Verkäufern erwarten, Hilfe bei ihrer Entscheidung zu bekommen, sich wohl zu fühlen und nicht in ihrer Entscheidung beeinflusst zu werden (vgl. Pieters/Bottschen/Thelen 1998, S. 763f.), ist als Konsequenz anzunehmen, dass die Beratungszufriedenheit der Kunden mit der Entscheidungsunterstützung, dem Aufbau eines positiven Gesprächsklimas und der Vermeidung von Abschlussdruck steigt.

Die vorgetragene Argumentation wird durch zahlreiche Befunde gestützt (vgl. z.B. Goff et al. 1997, S. 178; Haas 2006b; Menon/Dubé 2000; Niebisch 1993, S. 302, S. 312, S. 380; Stock/Hoyer 2005, S. 539). Damit werden folgende Hypothesen formuliert:

$\mathrm{H}_{1}$ : Entscheidungsunterstützung beeinflusst Beratungszufriedenheit positiv.

$\mathrm{H}_{2}$ : Aufbau eines positiven Gesprächsklimas beeinflusst Beratungszufriedenheit positiv.

$\mathrm{H}_{3}$ : Vermeidung von Abschlussdruck beeinflusst Beratungszufriedenheit positiv.

Weiterhin wird unterstellt, dass Beratungszufriedenheit einen positiven Einfluss auf die Kaufentscheidung der Kunden ausübt. Diese Annahme wird durch die Risikotheorie begründet (vgl. Bauer 1967): Aus risikotheoretischer Sicht verhindert bei Kunden, die eine Beratung in Anspruch nehmen, das als zu hoch empfundene Risiko eine unmittelbare Kaufentscheidung (vgl. Grunert 1978; Olshavsky 1973; Roselius 1971). Diese Kunden treffen ihre Kaufentscheidung erst dann, wenn es ihnen gelungen ist, das Risiko auf ein 
für sie akzeptables Niveau zu reduzieren. Eine Möglichkeit zu einer solchen Risikoreduktion besteht darin, dass die Kunden sich bei dem Berater zu einem Kauf entscheiden, mit dessen Beratung sie zufrieden sind. Insofern ist zu erwarten, dass die Wahrscheinlichkeit einer Kaufentscheidung mit steigender Beratungszufriedenheit zunimmt.

Verschiedentlich nachgewiesene Zusammenhänge zwischen verkäuferbezogenen Zufriedenheitsurteilen und der Kaufentscheidung der Kunden stützen diese Argumentation (vgl. Evans et al. 2000, S. 521f.; Niebisch 1993, S. 320; Verhoef/Franses/Donkers 2002, S. 130). Als Konsequenz wird folgende Hypothese aufgestellt:

$\mathrm{H}_{4}$ : Beratungszufriedenheit beeinflusst die Kaufentscheidung positiv.

\subsection{Fundierung der Hypothese zum direkten Effekt}

Als direkter Effekt wird ein nicht-linearer, umgekehrt u-förmiger, Einfluss der Vermeidung von Abschlussdruck auf die Kaufentscheidung unterstellt.

Der unterstellte Effekt beruht auf zwei Teileffekten: Der erste Teileffekt besteht in einem negativen Teileffekt progressiver Art der Vermeidung von Abschlussdruck. Denn es wird unterstellt, dass eine nicht vollständige Vermeidung von Abschlussdruck - also ein gewisser Aufbau von Abschlussdruck - zu einer deutlichen Erhöhung der Kaufwahrscheinlichkeit führt, indem der Verkäufer dadurch der kundenseitigen Tendenz entgegenwirkt, sich - sogar angesichts eines an sich vorteilhaften Produkts - nicht zu einem Kauf zu entscheiden. Dieses Verhalten geht darauf zurück, dass Menschen in mit Risiko behafteten Entscheidungssituationen oft dazu neigen, den Status Quo zu bewahren statt eine Veränderung herbeizuführen, selbst wenn die Konsequenzen einer Veränderung (objektiv) vorteilhaft sind (vgl. Samuelson/Zeckhauser 1988). Diese Neigung lässt sich durch die Abneigung, die Entscheidung später zu bedauern, und die im Vergleich zu den möglichen Gewinnen höhere Gewichtung der mit der Entscheidung verbundenen Verluste im Sinne der Prospect Theorie erklären (vgl. Schweitzer 1994, S. 458f.). Die Neigung zur Bewahrung des Status Quo wird verstärkt, wenn mehrere Produktalternativen als Entscheidungsoptionen existieren (vgl. Kempf/Ruenzi 2006; Samuelson/ Zeckhauser 1988; Tversky/Shafir 1992), wie es in Verkaufsgesprächen vorwiegend der Fall ist.

Als zweiter Teileffekt wird ein positiver Effekt degressiver Art der Vermeidung von Abschlussdruck unterstellt. Denn es wird unterstellt, dass eine nur geringe Vermeidung von Abschlussdruck - also ein starker Aufbau von Abschlussdruck - zu hohen Widerständen gegen einen Kauf und damit zu einem deutlichen Herabsetzen der Kaufwahrscheinlichkeit führt, während eine starke Vermeidung von Abschlussdruck keine Wirkungen auf den Kaufwiderstand hervorruft. Dieser Teileffekt wird durch die Reaktanztheorie gestützt, gemäß der das Einschränken der erwarteten Wahlfreiheit einer Person dazu führt, dass diese sich der Einengung widersetzt (vgl. Brehm 1966; Brehm/Brehm 1981). Während eine gewisse Einschränkung der von den Kunden erwarteten freien Entscheidung über Kauf oder Nicht-Kauf durch eine nicht vollständige Vermeidung von Abschlussdruck keine Wirkungen hervorruft, werden die dadurch ausgelösten Widerstände gegen den Kauf nach Erreichen einer bestimmten Schwelle um so stärker (vgl. Bateson 1985, S. 71; Kroeber-Riel/Weinberg 2003, S. 207). 
Die beiden Teileffekte führen insgesamt zu einem umgekehrt u-förmigen Gesamteffekt der Vermeidung von Abschlussdruck. Obwohl auf der konzeptionellen Ebene die Annahme eines solchen Funktionsverlaufs in der Literatur verschiedentlich zu erkennen ist (vgl. Brown 1990, S. 18f.; Hawes/Strong/Winick 1996, S. 350), wurde bisher kein nichtlinearer Zusammenhang zwischen abschlussorientiertem Verkäuferverhalten und Kaufentscheidung der Kunden überprüft. Gleichwohl weisen Studien sowohl positive als auch negative Effekte verschiedener Verkaufsabschlusstechniken nach und stützen so die vorgetragene Argumentation (vgl. DelVecchio et al. 2004, S. 867f.; McFarland/Challagalla/ Shervani 2006, S. 110). Damit lautet die Hypothese:

$\mathrm{H}_{5}$ : Die Vermeidung von Abschlussdruck beeinflusst die Kaufentscheidung zunächst positiv und dann negativ.

Die aufgestellten Hypothesen werden nachfolgend empirisch geprüft.

\section{Empirische Untersuchung}

\subsection{Datenerhebung und Datengrundlage}

Da das Verkäuferverhalten nach herrschender Meinung aus Sicht der Kunden zu erfassen ist (vgl. z. B. Brady/Cronin 2001, S. 244; Michaels/Day 1985, S. 443; Stock 2002, S. 61), bestand das Forschungsdesign zur Datenerhebung darin, dass Personen, die sich im Handel über Bekleidung oder Elektrogeräte beraten ließen, direkt im Anschluss an deren Verkaufsinteraktion mittels eines Fragebogens persönlich befragt wurden. Die Kaufentscheidung der Kunden wurde dabei von den Interviewern beobachtet. Es ergaben sich keine Abweichungen zu den Angaben der Kunden im Fragebogen.

Während die Inanspruchnahme von Beratung seitens der Kunden zeigte, dass es sich für diese um keine habitualisierte Kaufentscheidung handelte, wurde mit der Berücksichtigung von Verkaufsinteraktionen aus zwei unterschiedlichen Produktkategorien eine größere Reichweite der Ergebnisse angestrebt. Die Auswahl der Produktkategorien und ihrer Produkte erfolgte auf Basis von Befunden, die vergleichbare Kundenerwartungen an den Verkäufer - und somit einen über die beiden Produktkategorien vergleichbaren Wirkungsmechanismus des kundenorientierten Verkaufens - nahe legten (vgl. Weiber/ Adler 1995, S. 107ff.). ${ }^{2}$ Die dortigen Befunde zeigen zudem, dass die hier berücksichtigten Produktkategorien ein vergleichsweise geringes Risiko aufweisen. Entsprechend war davon auszugehen, dass der für leichten Abschlussdruck argumentierte „Kaufimpuls“ nicht durch Kaufwiderstände überlagert wird, die auf ein höheres Produktrisiko (z.B. bei Vertrauensgütern) zurückgehen.

Insgesamt wurden 203 Konsumenten zu jeweils unterschiedlichen Verkäufern befragt. $50,2 \%$ der Befragten waren männlich, 49,8\% der Befragten weiblich. Das Alter der Befragten reichte von 17 bis 82 Jahren mit einem Mittelwert bei 43,4 Jahren. Damit unterscheidet sich die Stichprobe nicht von der Bevölkerung in Deutschland (vgl. CIA 2006; Statistisches Bundesamt 2006). Als höchsten Bildungsabschluss gaben von den Befragten 23,8\% den Hauptschulabschluss an, 29,7\% Mittlere Reife, 20,3\% Abitur und 26,2\% einen Hochschulabschluss. 
Als Ergebnis standen die Daten von 203 Verkaufsinteraktionen für die Auswertung zur Verfügung. 21,2\% davon bezogen sich auf Bekleidung (Herrenoberbekleidung, Damenoberbekleidung), 78,8\% auf elektrotechnische und elektronische Produkte (Computer, Foto, braune und weiße Ware). Mit Blick auf die Art der Geschäfte wurden 5,5\% der Interaktionen in Elektro-Fachgeschäften bzw. Boutiquen geführt, 11,3\% in Warenhäusern und $83,2 \%$ in Elektro- bzw. Bekleidungsfachmärkten. Dabei war nicht davon auszugehen, dass die Betriebsform einen Einfluss auf die zu untersuchenden Zusammenhänge hat. Denn die befragten Personen hatten die Betriebsform für ihre Beratung selbst bestimmt. Dafür lassen Befunde in der Literatur vermuten, dass Kunden vergleichbare Erwartungen an die Beratung aufweisen (vgl. Hisrich/Dornoff/Kernan 1972). ${ }^{3}$

\subsection{Konstruktmessung}

Für die Variable Kaufentscheidung wurde Kauf vs. Nicht-Kauf erhoben. Die anderen Konstrukte wurden in Anlehnung an einschlägige Skalen durch siebenstufige Multi-ItemMessungen erfasst (1=,stimme gar nicht $\mathrm{zu}^{\text {“; }} 7=$,stimme völlig zu“) (vgl. Donavan/ Brown/Mowen 2004; Lee/Dubinsky 2003; Saxe/Weitz 1982; Van Dolen et al. 2002). Die Items der Konstrukte inkl. ihrer statistischen Kennwerte finden sich im Anhang.

Um die Eignung der Items sicherzustellen, wurden diese vor Beginn der Studie von sechs Personen, die sich im Hinblick auf verschiedene Charakteristika (Geschlecht, Alter, Ausbildung, Beruf, Erfahrung mit Datenerhebungen) unterschieden, hinsichtlich allgemeiner Anforderungen an die Itemformulierung bei Datenerhebungen geprüft (vgl. DeVellis 1991, S. 57ff.; Niebisch 1993, S. 236ff.). Die Ergebnisse ließen auf keinen Änderungsbedarf schließen. Im Fragebogen waren die Items nicht pro Konstrukt, sondern vermischt angeordnet.

Um die Reliabilität und Validität der Konstruktmessung zu prüfen, wurde neben der exploratorischen Faktorenanalyse insbesondere die Methode der konfirmatorischen Faktorenanalyse mit LISREL 8.71 angewendet. Wie in der Literatur üblich (vgl. z.B. Bettencourt/Brown/MacKenzie 2005, S. 149), wurde dazu ein konfirmatorisches Faktorenmodell der durch Multi-Item-Messungen erfassten Konstrukte mit den Kovarianzen des Messinstruments als Input geschätzt. Die Analysen bestätigten die Güte des Messmodells. Da im Zuge der späteren Wirkungsanalysen vier Ausreißer identifiziert und aus dem Datensatz entfernt wurden (vgl. Abschnitt 5), wurden die Analysen für den reduzierten Datensatz $(n=199)$ wiederholt. Der verbleibende Stichprobenumfang erfüllt die an diese Analyse gestellte Anforderung der Literatur (vgl. Bearden/Sharma/Teel 1982, S. 429). Die nachfolgend berichteten Werte beziehen sich auf den reduzierten Datensatz.

Die Ergebnisse der konfirmatorischen Faktorenanalyse weisen auf die Güte des Messmodells ( $\chi^{2}$-Wert: 223, 92; df: 98) hin. So lauten der Goodness of Fit Index (GFI) 0,981, der Adjusted Goodness of Fit Index (AGFI) 0,973 und der Root Mean Square Error of Approximation (RMSEA) 0,08. Die ersten beiden Werte überschreiten die in der Literatur geforderten Mindestwerte von jeweils 0,9 deutlich, und auch der RMSEA-Wert weist auf eine akzeptable Modellanpassung hin (vgl. Homburg/Baumgartner 1995, S. 167f.; Homburg/Pflesser 1999, S. 430). Wie man dem Anhang entnehmen kann, unterschreiten die Faktorladungen der einzelnen Messgrößen in keinem Fall den in der Literatur für den vorliegenden Stichprobenumfang genannten Schwellenwert von 0,6 (vgl. Balderjahn 
Tab. 1. Informationen zur Konstruktmessung

\begin{tabular}{|l|c|c|c|c|}
\hline Faktor & Itemanzahl & Alpha & FR & DEV \\
\hline Entscheidungsunterstützung & 4 & 0,81 & 0,82 & 0,53 \\
\hline Aufbau positives Gesprächsklima & 5 & 0,82 & 0,83 & 0,51 \\
\hline Vermeidung Abschlussdruck & 4 & 0,85 & 0,87 & 0,64 \\
\hline Beratungszufriedenheit & 3 & 0,92 & 0,92 & 0,79 \\
\hline
\end{tabular}

Alpha: Cronbachs Alpha; FR: Faktorreliabilität; DEV: durchschnittlich erfasste Varianz

1986, S. 117). Zudem zeigten die Analyseergebnisse, dass alle Faktorladungen auf dem 0,01-Niveau signifikant sind. Darüber hinaus kann man Tabelle 1 entnehmen, dass die Anforderungen der Literatur an das Cronbachs Alpha $(>0,7)$, die Faktorreliabilität $(>0,6)$ und die durchschnittlich erfasste Varianz $(>0,5)$ ausnahmslos erfüllt werden (vgl. Homburg/Pflesser 1999, S. 430; Nunnally 1978, S. 245).

Die Prüfung der Diskriminanzvalidität der drei Dimensionen erfolgte durch das vergleichsweise strenge Fornell/Larcker-Kriterium (vgl. Fornell/Larcker 1981, S. 46). Danach liegt Diskriminanzvalidität zwischen zwei Faktoren vor, wenn die durchschnittlich erfasste Varianz (DEV) jedes dieser Faktoren größer ist als die quadrierte Korrelation zwischen diesen beiden Faktoren. Dies war für jeden Fall gegeben. Der größte Wert für die quadrierte Korrelation ergab sich dabei mit 0,48 zwischen Entscheidungsunterstützung $(\mathrm{DEV}=0,53)$ und dem Aufbau eines positiven Gesprächsklimas $(\mathrm{DEV}=0,51){ }^{4}$

\section{Ergebnisse der Untersuchung}

Die postulierten Wirkungszusammenhänge wurden mittels der Methoden der Regressionsanalyse und der logistischen Regression untersucht (vgl. Albers/Skiera 1999; Hosmer/ Lemeshow 2000; Krafft 1997). Die Konstrukte gingen in Form des arithmetischen Mittels über die konstruktspezifischen Indikatoren in die Analysen ein. Zur Prüfung des unterstellten nicht-linearen Zusammenhangs zwischen Vermeidung von Abschlussdruck und Kaufentscheidung wurde das Quadrat von Vermeidung von Abschlussdruck als eigenständige Variable berechnet. Wie in der Literatur vorgeschlagen, wurde bei den nachfolgenden Analysen zuvor ein Mean Centering der Regressoren vorgenommen, um das Problem der skalierungsbedingten Multikollinearität zu beheben (vgl. Cohen et al. 2003, S. 203).

Im Rahmen der Analysen wurde der Datensatz zunächst auf mögliche Ausreißer untersucht. Die entsprechenden diagnostischen Analysen (vgl. Cohen et al. 2003, S. 394ff.; Hosmer/Lemeshow 2000, S. 167ff.) führten bei der Regressionsanalyse und bei der logistischen Regression jeweils zur Identifizierung von zwei Fällen. Da die inhaltliche Prüfung dieser Fälle deren Einstufung als Ausreißer gerechtfertigt erscheinen ließ, wurden diese vier Fälle aus dem Datensatz entfernt. Alle folgenden Analysen basieren somit auf 199 Datensätzen.

In den Hypothesen $\mathrm{H}_{1}, \mathrm{H}_{2}, \mathrm{H}_{3}$ und $\mathrm{H}_{4}$ wurde unterstellt, dass Beratungszufriedenheit die Wirkungen von Entscheidungsunterstützung, Aufbau eines positiven Gesprächsklimas und Vermeidung von Abschlussdruck auf die Kaufentscheidung der Kunden mediiert. 
Tab. 2. Analyseergebnisse

\begin{tabular}{|c|c|c|c|c|c|}
\hline $\begin{array}{l}\text { Modell } \\
\text { abhängige Variable } \\
\text { unabhängige Variable }\end{array}$ & $\begin{array}{c}1 \\
\text { Beratungs- } \\
\text { zufriedenheit }^{\mathrm{a}}\end{array}$ & $\underset{\text { Kauf }^{\mathbf{b}}}{\mathbf{2 a}}$ & $\begin{array}{c}2 b \\
\text { Kauf }^{b}\end{array}$ & $\begin{array}{c}\text { 3a } \\
\text { Kauf }^{b}\end{array}$ & $\begin{array}{c}\text { 3b } \\
\text { Kauf }^{\mathbf{b}}\end{array}$ \\
\hline $\begin{array}{l}\text { Entscheidungs- } \\
\text { unterstützung }\end{array}$ & $\begin{array}{c}0,343 * * * \\
(6,476)\end{array}$ & $\begin{array}{c}0,200 / 1,221 * \\
(3,114)\end{array}$ & $\begin{array}{l}0,077 / 1,080 \\
(0,367)\end{array}$ & $\begin{array}{l}0,062 / 1,064 \\
(0,238)\end{array}$ & $\begin{array}{c}0,071 / 1,073 \\
(0,324)\end{array}$ \\
\hline $\begin{array}{l}\text { Aufbau positives } \\
\text { Gesprächsklima }\end{array}$ & $\begin{array}{c}0,477 * * * \\
(9,033)\end{array}$ & $\begin{array}{c}-0,120 / 0,887 \\
(0,849)\end{array}$ & $\begin{array}{c}-0,341 / 0,711^{* *} \\
(4,272)\end{array}$ & $\begin{array}{c}-0,329 / 0,719^{* *} \\
(3,954)\end{array}$ & $\begin{array}{c}-0,325 / 0,723^{* *} \\
(3,880)\end{array}$ \\
\hline $\begin{array}{l}\text { Vermeidung Abschluss- } \\
\text { druck }\end{array}$ & $\begin{array}{c}0,362 * * * \\
(8,335)\end{array}$ & $\begin{array}{c}0,363 / 1,437 * * * \\
(8,920)\end{array}$ & $\begin{array}{l}0,211 / 1,234 \\
(2,240)\end{array}$ & $\begin{array}{c}-0,066 / 0,936 \\
(0,085)\end{array}$ & \\
\hline Beratungszufriedenheit & & & $\begin{array}{c}0,427 / 1,533^{* * *} \\
(5,441)\end{array}$ & $\begin{array}{c}0,417 / 1,517 * * \\
(5,068)\end{array}$ & $\begin{array}{c}0,404 / 1,497 * * \\
(5,058)\end{array}$ \\
\hline $\begin{array}{l}\text { Vermeidung Abschluss- } \\
\text { druck quadriert }\end{array}$ & & & & $\begin{array}{c}-0,130 / 0,878^{*} \\
(2,139)\end{array}$ & $\begin{array}{c}-0,111 / 0,895^{* *} \\
(3,518)\end{array}$ \\
\hline $\begin{array}{l}\text { Korr. } \mathrm{R}^{2} \\
\text { F-Wert }\end{array}$ & $\begin{array}{c}0,629 \\
112,75^{* * *}\end{array}$ & & & & \\
\hline $\begin{array}{l}\text { Nagelkerkes } \mathrm{R}^{2} / \\
\text { McFaddens } \mathrm{R}^{2} \\
\chi^{2} \text {-Wert }\end{array}$ & & $\begin{array}{c}0,080 / 0,046 \\
11,97 * * *\end{array}$ & $\begin{array}{c}0,117 / 0,068 \\
17,66^{* * *}\end{array}$ & $\begin{array}{c}0,133 / 0,078 \\
20,24 * * *\end{array}$ & $\begin{array}{c}0,133 / 0,078 \\
20,15 * * *\end{array}$ \\
\hline
\end{tabular}

${ }^{a}$ : standardisierte Koeffizienten; ***: signifikant auf dem 0,01-Niveau; t-Werte in Klammern

b: Referenzkategorie: Kauf=nein; Werte: Koeffizient/ odds ratio; ***: signifikant auf dem 0,01-Niveau; **: signifikant auf dem 0,05-Niveau; *: signifikant auf dem 0,1-Niveau; Wald-Statistik-Werte in Klammern

Analog zu entsprechendem Vorgehen in der Literatur (vgl. z.B. Licata et al. 2003, 262f.) wurden diese Hypothesen simultan in Anlehnung an das von Baron/Kenny (1986) vorgeschlagene Vorgehen zum Test auf mediierende Effekte geprüft. Tabelle 2 stellt die Ergebnisse der Analysen dar.

In einem ersten Schritt wurde eine Regressionsanalyse mit Entscheidungsunterstützung, Aufbau eines positiven Gesprächsklimas und Vermeidung von Abschlussdruck als unabhängigen Variablen und Beratungszufriedenheit als abhängiger Variable gerechnet (s. Modell 1).

Hinsichtlich der diversen Annahmen der Methode der Regressionsanalyse (vgl. Kutner/Nachtsheim/Neter 2004, S. 9f.) zeigte sich für die Analyse keine ernsthafte Prämissenverletzung. Speziell gibt es keine Anzeichen für ernsthafte Multikollinearität, da der maximale Wert des Variance Inflation Factor in den Analysen mit 1,494 deutlich unter dem in der Literatur genannten Schwellenwert von 10 liegt (vgl. Kutner/Nachtsheim/ Neter 2004, S. 409). Angemerkt sei aber, dass im Fall der Variablen Vermeidung von Abschlussdruck die Prüfung auf Normalverteilung knapp am 0,05-Niveau scheiterte. Die inferenzstatistische Absicherung der Ergebnisse der Regressionsanalyse gegenüber geringfügigen Verletzungen der Annahmen ist allerdings relativ robust (vgl. z.B. Bortz 1999, S. 184; Cohen et al. 2003, S. 96). Dennoch gilt es, diese Einschränkung bei der weiteren Ergebnisdarstellung zu berücksichtigen.

Die Ergebnisse zeigen einen positiven Einfluss der Entscheidungsunterstützung, des Aufbaus eines positiven Gesprächsklimas und der Vermeidung von Abschlussdruck und bestätigen so die Hypothesen $\mathrm{H}_{1}, \mathrm{H}_{2}$ und $\mathrm{H}_{3}$. 
In einem nächsten Schritt wurde der unterstellte mediierende Effekt der Beratungszufriedenheit geprüft, indem die Modelle $2 \mathrm{a}$ und $2 \mathrm{~b}$ verglichen wurden (s. Tabelle 2). In Modell 2a wird eine logistische Regression mit Entscheidungsunterstützung, Aufbau eines positiven Gesprächsklimas und Vermeidung von Abschlussdruck als unabhängigen Variablen und Kaufentscheidung als abhängiger Variable gerechnet. In das Modell 2b wird im Vergleich zu Modell 2a Beratungszufriedenheit als zusätzliche unabhängige Variable neben den Dimensionen des kundenorientierten Verkaufens integriert. Bei der Prüfung der entsprechenden Annahmen der logistischen Regression (vgl. Krafft 1999, S. 250) zeigte sich für die beiden Modelle keine ernsthafte Prämissenverletzung.

Die Ergebnisse zeigen einen positiven Zusammenhang zwischen Beratungszufriedenheit und Kaufentscheidung (s. Tabelle 2, Modell 2b). Dadurch wird Hypothese $\mathrm{H}_{4}$ bestätigt. Zudem zeigt sich in Modell 2a ein signifikanter Zusammenhang zwischen Vermeidung von Abschlussdruck und Kaufentscheidung. Dieser wird nicht signifikant, wenn zusätzlich Beratungszufriedenheit im Modell berücksichtigt wird (s. Tabelle 2, Modell 2b). Somit wird der Zusammenhang zwischen Vermeidung von Abschlussdruck und Kaufentscheidung vollständig durch Beratungszufriedenheit mediiert. Auch die Stärke des Zusammenhangs zwischen Entscheidungsunterstützung und Kaufentscheidung verringert sich beim Übergang von Modell $2 \mathrm{a}$ zu $2 \mathrm{~b}$ deutlich. Angesichts der positiven $\mathrm{Zu}-$ sammenhänge zwischen Entscheidungsunterstützung und Beratungszufriedenheit sowie zwischen Beratungszufriedenheit und Kaufentscheidung deutet dieser Befund ebenfalls darauf hin, dass der Zusammenhang zwischen Entscheidungsunterstützung und Kaufentscheidung vollständig durch Beratungszufriedenheit mediiert wird. Der entsprechende Test (vgl. Kenny/Kashy/Bolger 1998, S. 260) weist den mediierten Effekt als signifikant aus (0,05-Niveau). ${ }^{5}$ Für den Aufbau eines positiven Gesprächsklimas zeigen die Ergebnisse, dass der Einfluss dieser Variablen auf die Kaufentscheidung nur teilweise durch die Beratungszufriedenheit mediiert wird. Denn bei der Berücksichtigung von Beratungszufriedenheit als unabhängige Variable zeigt sich ein direkter Effekt von Aufbau eines positiven Gesprächsklimas auf die Kaufentscheidung (s. Tabelle 2, Modell 2b). Zudem weisen die Ergebnisse einen gegenläufigen Effekt des Aufbaus eines positiven Gesprächsklimas auf die Kaufentscheidung - positiv über die Beratungszufriedenheit und negativ direkt nach. ${ }^{6}$ Der nachgewiesene negative direkte Effekt bestätigt entsprechende empirische Hinweise auf einen derartigen Effekt in der Literatur (vgl. Evans et al. 2000, S. 522).

Schließlich wurde Hypothese $\mathrm{H}_{5}$ geprüft, indem eine logistische Regression mit Entscheidungsunterstützung, Aufbau eines positiven Gesprächsklimas, Vermeidung von Abschlussdruck, Beratungszufriedenheit und dem Quadrat von Vermeidung von Abschlussdruck als unabhängigen Variablen und Kaufentscheidung als abhängiger Variable gerechnet wurde (s. Tabelle 2, Modell 3a).

Die Prüfung der Annahmen der logistischen Regression (vgl. Krafft 1999, S. 250) zeigte eine hohe Korrelation zwischen der Vermeidung von Abschlussdruck und dem Quadrat dieser Variablen $(\mathrm{r}=-0,83){ }^{7}$ Diese kann problematisch sein, da dadurch für den Zusammenhang zwischen dem Quadrat der Vermeidung von Abschlussdruck und Kaufentscheidung ein zu großer Standardfehler geschätzt werden kann und als Folge ein vorhandener Zusammenhang zwischen diesen Variablen als nicht signifikant ausgewiesen werden kann (vgl. Hosmer/Lemeshow 2000, S. 141). Daraus würde die Gefahr einer Ablehnung der Hypothese H5 trotz ,in Wahrheit“" bestehenden Zusammenhangs resultieren. 
Wie in der Literatur empfohlen (vgl. Hosmer/Lemeshow 2000, S. 97), wurde zur Überprüfung dieses Sachverhalts die nicht-signifikante Variable Vermeidung von Abschlussdruck aus dem Modell entfernt und das resultierende Modell neu geschätzt (s. Tabelle 2, Modell 3b). Während sich die Gütemaße des Gesamtmodells zwischen den Modellen 3a und $3 \mathrm{~b}$ nicht sowie die Koeffizienten und Standardfehler der anderen Variablen kaum veränderten, zeigte sich, wie erwartet, dass der Standardfehler des Quadratterms im Modell mit der Variablen Vermeidung von Abschlussdruck mit einem um 50\% höheren Wert deutlich über dem Standardfehler des Quadratterms in Modell 3b lag.

An dieser Stelle stellt sich die Frage, ob das Entfernen der Variablen Vermeidung von Abschlussdruck aus dem Modell auch zulässig ist. Diesbezüglich wird traditionell in der Literatur argumentiert, dass es bei Modellen mit Termen höherer Ordnung (z.B. Quadratterme oder Produktterme, wie bei der moderierten Regression) nötig ist, alle Variablen in das Modell aufzunehmen, aus denen die Terme höherer Ordnung bestehen, um eine fehlerhafte Varianzzuordnung zu diesen Termen zu vermeiden (z.B. Cohen et al. 2003; Irvin/McClelland 2001). Solche Zuordnungseffekte können Gatignon/Vosgerau (2006) in ihrer Simulationsstudie jedoch nicht nachweisen. ${ }^{8}$ Zudem zeigen sie, dass insbesondere fehlerhaft spezifizierte Modelle zu Ergebnisverzerrungen bezüglich des Terms höherer Ordnung führen, und folgern, dass Effekte der einzelnen Variablen dieses Terms nicht ,automatisch“, sondern nur im Falle einer theoretischen Begründung zusätzlich in das Modell aufgenommen werden sollten.

Im vorliegenden Fall ist eine theoretische Argumentation für den Quadratterm erkennbar, nicht aber für einen zusätzlichen linearen Effekt der Vermeidung von Abschlussdruck. In diesem Sinne stützen die Befunde zu Modell 3a die Annahme eines nicht existierenden Effekts der Vermeidung von Abschlussdruck. Auch stimmt die Veränderung des Signifikanzniveaus für den Effekt des Quadratterms beim Übergang von Modell 3a zu 3b mit dem von Gatignon/Vosgerau (2006) identifizierten Muster beim Wechsel von einem fehlzu einem korrekt spezifizierten Modell überein. Insgesamt lässt sich daraus eine Ergebnisverzerrung bezüglich des Quadratterms im Falle der Integration der Variablen Vermeidung von Abschlussdruck in das Modell folgern. Als Konsequenz stellt das Modell ohne diese Variable die Basis für die folgende Hypothesenprüfung dar (s. Tabelle 2, Modell 3b).

Mit Blick auf die Güte des Modells sind die Pseudo- $\mathrm{R}^{2}$-Werte zwar gering, sie stimmen aber in der Höhe mit bisherigen Befunden in der Literatur zu einem nur schwachen Zusammenhang zwischen verkäuferbezogenen Zufriedenheitsurteilen und der Kaufentscheidung der Kunden überein (vgl. Evans et al. 2000, S. 522; Niebisch 1993, S. 320; Verhoef/ Franses/Donkers 2002, S. 130). Der Anteil der korrekt klassifizierten Elemente liegt mit dem Wert von 72\% über dem Proportional Chance-Kriterium von 52\% und erfüllt damit die Anforderungen der Literatur (vgl. Krafft 1999, S. 248; Morrison 1969, S. 158).

Hypothese $\mathrm{H}_{5}$, die einen nicht-linearen Einfluss der Vermeidung von Abschlussdruck auf die Kaufentscheidung unterstellt, wird durch die Ergebnisse bestätigt. Denn das negative Vorzeichen des quadratischen Terms bestätigt den vermuteten Funktionsverlauf. Zudem zeigt sich dieser Zusammenhang als signifikant. ${ }^{9}$ Dieses Ergebnis zeigt, dass ein direkter nichtlinearer Effekt der Vermeidung von Abschlussdruck auf die Kaufentscheidung besteht.

Um den Wirkmechanismus des kundenorientierten Verkaufens auf die kurzfristige Kaufentscheidung zusätzlich auszuleuchten, wurde ex post über die unterstellten Hypothesen hinaus zudem geprüft, ob sich die Einflussstärke der Dimensionen des kundenorientierten Verkaufens auf die Beratungszufriedenheit unterscheidet. Der entsprechende 
Test (vgl. Förster/Rönz 1979, S. 218f.) zeigte, dass der Einfluss des Aufbaus eines positiven Gesprächsklimas stärker als der Einfluss der Entscheidungsunterstützung ist $(0,05$ Niveau). Dieses Ergebnis stimmt mit Befunden in der Literatur überein, nach denen für Konsumenten die sozio-emotionalen Aspekte von Verkaufsgesprächen bedeutsamer sind als die sachlichen (vgl. Crosby/Evans/Cowles 1990, S. 76; Pieters/Bottschen/Thelen 1998, S. 764f.). Die Einflussunterschiede zwischen Entscheidungsunterstützung und Vermeidung von Abschlussdruck sowie zwischen Aufbau eines positiven Klimas und Vermeidung von Abschlussdruck zeigten sich dagegen als nicht signifikant. ${ }^{10}$ Die Ergebnisse bedeuten, dass die sozio-emotionale Facette des kundenorientierten Verkaufens die Beratungszufriedenheit stärker beeinflusst als die aufgabenbezogene Facette. Zugleich entfällt damit auf die Entscheidungsunterstützung weniger als ein Drittel des Gesamteinflusses des kundenorientierten Verkaufens auf die Beratungszufriedenheit.

\section{Schlussbetrachtung}

Die Frage nach dem Einfluss der Kundenorientierung von Verkäufern auf deren Verkaufsergebnis besitzt eine hohe praktische Relevanz. Auf Basis der vorhandenen Befunde konnte die Wissenschaft einen Einfluss bisher nicht bestätigen. Vor diesem Hintergrund wurde im vorliegenden Beitrag analysiert, wie das kundenorientierte Verkaufen in seinen Dimensionen auf die in einer Verkaufsinteraktion anstehende Kaufentscheidung der Kunden wirkt.

Aus wissenschaftlicher Sicht liefern die Analysen erstmals differenzierte Erkenntnisse über den Wirkmechanismus des kundenorientierten Verkaufens auf die Kaufentscheidung von Kunden: Die Ergebnisse belegen den indirekten Effekt über die Beratungszufriedenheit. Daneben zeigten sich ein direkter umgekehrt u-förmiger Effekt der Vermeidung von Abschlussdruck sowie ein direkter negativer Effekt des Aufbaus eines positiven Gesprächsklimas auf die Kaufentscheidung. Zudem geht aus den Ergebnissen hervor, dass die Beratungszufriedenheit nur unterdurchschnittlich durch die Entscheidungsunterstützung beeinflusst wird. Insgesamt zeigen die Ergebnisse nicht nur die Notwendigkeit zu einer stärkeren Berücksichtigung des Kaufverhaltens als Erfolgsgröße, sondern bestätigen auch die Zweckmäßigkeit der Analyse des kundenorientierten Verkaufens auf der Ebene der Dimensionen dieses Konstrukts. Der nachgewiesene nicht-lineare Effekt der Vermeidung von Abschlussdruck impliziert dabei, dass ein Verkaufsabschluss wahrscheinlicher ist, wenn ein gewisser Abschlussdruck aufgebaut wird, als wenn auf solchen Druck vollständig verzichtet wird. Letzteres wird in der Literatur von kundenorientierten Verkäufern gemeinhin gefordert (vgl. z.B. Saxe/Weitz 1982), führt insofern aber unternehmensseitig zu Nachteilen bezüglich der Verkaufsabschlüsse. In diesem Sinne kann die Kundenorientierung von Verkäufern folglich auch zu hoch sein.

Für die Unternehmenspraxis unterstreichen die Ergebnisse die Erfolgsrelevanz einer auf Produkt-Know-how und Sozialkompetenz aufbauenden Beratung. Daraus folgt, dass unternehmensseitige Investitionen in die Produkt- und Beratungskompetenz ihrer Mitarbeiter nicht nur lohnend, sondern auch notwendig sind. Zudem legen die Ergebnisse nahe, dass Verkäufer bei den Kunden durch „,sanften Druck“ den Kaufimpuls auslösen können. Daraus sollte nicht die Vorteilhaftigkeit des Hard Selling geschlossen werden. Denn der Abschlussdruck übersteigt schnell das zweckmäßige Niveau und führt dann zu deutlich 
negativen Kundenempfindungen, die letztlich auch einen Verkaufsabschluss unmöglich machen. Insgesamt werden somit Verkaufs- und Abschlusstechniken bedeutsam, die die Kundenentscheidung ,anstoßen“, ohne dass der Kunde dies als Beeinflussung wahrnimmt. Schließlich wird deutlich, dass die Beratungszufriedenheit, die für die Loyalität der Kunden bedeutsam ist (vgl. Hennig-Thurau 2004), insbesondere vom auf die Verkäufer-Kunde-Beziehung bezogenen Verkäuferverhalten abhängt. Damit ist die Gestaltung der zwischenmenschlichen Beziehung zum Kunden ein eigenständiges Aufgabenfeld des Verkäufers - und damit ein integrativer Bestandteil der Verkaufsaufgabe. Dabei belegen die Ergebnisse zu den gegensätzlichen Wirkungen dieses Verhaltens ein seit langem diskutiertes Dilemma der Verkäufer (vgl. Brown 1990, S. 18). So müssen sie einerseits einen positiven Eindruck aufbauen, um erfolgreich zu sein. Andererseits ist dieses Verhalten den Kunden dadurch suspekt, dass die Verkäufer von einem solchen positiven Eindruck profitieren - und steht so der Kaufentscheidung der Kunden entgegen.

Mit Blick auf die weitere Forschung stimmt der geringe Zusammenhang zwischen Beratungszufriedenheit und Kaufentscheidung der Kunden zwar mit bisherigen Befunden in der Literatur überein, führt aber gleichzeitig zur Frage nach den Gründen dafür. Neben gewissen nicht auszuschließenden Habitualisierungseffekten (z.B. in Form geschäftsbezogenen Wiederkaufverhaltens) lassen weitere Ergebnisse der durchgeführten Analysen insbesondere die vorab bestehende Kaufabsicht der Kunden als Ursache vermuten. In der Praxis problematischer ist dabei der Fall der vorab fehlenden Kaufabsicht der Kunden, insbesondere in Form des Free Riding (vgl. Singley/Williams 1995). Entsprechend erscheinen Forschungsbemühungen zweckmäßig, die sich auf die Rolle der vor der Verkaufsinteraktion bestehenden Kaufabsicht und die Möglichkeiten ihrer Beeinflussung im Verkaufsgespräch konzentrieren.

Zudem stellt sich die Frage, wodurch sich der direkte negative Effekt des Aufbaus eines positiven Gesprächsklimas auf die Kaufentscheidung erklären lässt und inwiefern dieser von dritten Variablen abhängt. Diesbezüglich zeigen jüngere Forschungsergebnisse, dass Emotionen als „Informationen“ in die Urteilsbildung von Konsumenten eingehen und dabei von diesen besonders schnell verarbeitet werden (vgl. Pham et al. 2001; Schwarz 1990). Auch lässt die Literatur vermuten, dass derartige emotionale Informationen von Konsumenten in Abhängigkeit der Authentizität des Verkäufers und des kundenseitigen Vertrauens zum Verkäufer verarbeitet werden (vgl. Doney/Cannon 1997; Schaefer/Pettijohn 2006, S. 26). Entsprechend könnte der Effekt darauf zurückgehen, dass die im Rahmen der vorliegenden Studie befragten Konsumenten das Bemühen um den Kunden seitens der hier betrachteten Verkäufer (mehrheitlich) als gekünstelt und/ oder unglaubwürdig wahrgenommen haben.

Darüber hinaus gilt es zu klären, warum das sozio-emotionale Verkäuferverhalten einen stärkeren Einfluss auf die Beratungszufriedenheit ausübt als das aufgabenorientierte. Ein Erklärungsansatz basiert darauf, dass Kunden Verkäufern in erheblichem Maße misstrauen (vgl. Obermiller/Spangenberg 2000, S. 316). Als Folge würden Konsumenten das Verkäuferverhalten, das abzuschätzen hilft, ob der Verkäufer im Zuge seiner Beratung wirklich am Kunden interessiert ist (=Aufbau eines positiven Gesprächsklimas), stärker beachten als die Beratungsinhalte (=Entscheidungsunterstützung).

Die zwei zuletzt diskutierten Befunde führen zudem zur Frage nach der Reichweite der Ergebnisse. So ist es denkbar, dass der negative Effekt des Aufbaus eines positiven Ge- 
sprächsklimas auf die anstehende Kaufentscheidung davon abhängt, ob diese Kaufentscheidung in einer frühen oder einer späteren Phase der Verkäufer-Kunde-Beziehung ansteht. Denn das Misstrauen, das in einer frühen Phase aufgrund fehlender Informationen über den Verkäufer (noch) besteht, kann sich im Laufe der Zeit durch die Erfahrung mit dem Verkäufer auflösen. Aufgrund jüngster Befunde (vgl. Homburg/Koschate/Hoyer 2006) ist darüber hinaus eine Veränderung der hier gefundenen zufriedenheitsbezogenen Effektstärken von Entscheidungsunterstützung und Aufbau eines positiven Klimas im Zeitverlauf anzunehmen. Neben einer erinnerungsbedingten „Umgewichtung“ der Einflüsse (z.B. wenn sich vor allem die kompetente Beratung im Gedächtnis ,festsetzt“) oder veränderten Effektstärken infolge rückblickender Betrachtungen (z.B. wenn sich die inhaltliche Beratung als unzutreffend herausstellt) können sich die hier gefundenen Effektunterschiede zwischen den beiden Dimensionen und der Beratungszufriedenheit auch mit fortschreitender Reife der Verkäufer-Kunde-Beziehung verändern. Aus risikotheoretischer Sicht wäre durch den immer besseren Informationsstand über den Verkäufer insbesondere eine Verringerung des verkäuferbezogenen Risikos zu erwarten. Auf Basis der oben ausgeführten Überlegungen sollte sich damit die Effektstärke des Aufbaus eines positiven Gesprächsklimas reduzieren. Dies könnte die entsprechenden Ergebnisse von Boles/ Johnson/Barksdale (2000, S. 79) erklären. Gleichwohl könnten diese aber auch aus veränderten Effektstärken der Dimensionen im Business-to-Business-Bereich resultieren.

Des Weiteren kann sich eine andere Wirkung des abschlussbezogenen Verhaltens in Verkaufssituationen ergeben, in denen das Produktrisiko am Ende einer Beratung höher ist, wie es z.B. bei Vertrauensgütern denkbar ist. Denn dann kann es sein, dass ein leichter Impuls, wie er in den hier untersuchten, vergleichsweise risikoarmen Entscheidungssituationen für das Umsetzen einer Entscheidung ausreichend war, nicht mehr genügt, um die Entscheidung ,anzustoßen“. Angesichts des höheren Risikos wäre für solche Situationen zudem zu vermuten, dass die Wirkung des abschlussbezogenen Verhaltens von kundenseitigen Unterschieden im Entscheidungsverhalten unter Risiko beeinflusst wird (vgl. z.B. Stafford/Greer 1965).

Aus methodischer Sicht bietet die festgestellte nicht-symmetrische Verteilung der Variablen Vermeidung von Abschlussdruck und die daraus folgende hohe Korrelation zwischen dieser Variablen und dem daraus gebildeten Quadratterm einen Ansatzpunkt für zukünftige Forschung. Angesichts eines in der Praxis vorwiegend nur schwach aufgebauten Abschlussdrucks erscheint ein experimentelles Erhebungsdesign zweckmäßig, in dem die Vermeidung von Abschlussdruck manipuliert wird, um die Korrelation zwischen dieser Variablen und dem Quadratterm zu verringern (vgl. Cohen et al. 2003, S. 427).

Methodisch lässt auch das im vorliegenden Beitrag gewählte Vorgehen, das Verkäuferverhalten und die Beratungszufriedenheit bei derselben Person zu messen, Raum für weitere Forschung. Obwohl ein anderes Vorgehen in der Literatur als problematisch angesehen wird (vgl. Jaccard/King/Pomazal 1977; Kraus 1995; Michaels/Day 1985, S. 443; Stock 2002, S. 61) und diese Sichtweise durch aktuelle Befunde gestützt wird, die eine von den Kunden abweichende Beurteilung des eigenen Verhaltens durch die Verkäufer zeigen (vgl. Stock/Hoyer 2005, S. 544), lässt sich nicht ausschließen, dass durch das gewählte Vorgehen ein sog. Common-Method-Bias, etwa in Form von Ausstrahlungseffekten, entsteht (vgl. Podsakoff et al. 2003). Insofern könnte eine zukünftige Analyse die hier genutzte Methodik um die Beobachtung des Verkäuferverhaltens ergänzen. Dadurch lie- 
ßen sich Rückschlüsse auf einen eventuell vorhandenen Common-Method-Bias ziehen. Zudem wäre es dadurch möglich, Unterschiede zwischen dem von den Verkäufern objektiv an den Tag gelegten und dem von den Kunden subjektiv wahrgenommenen Verkäuferverhalten zu untersuchen.

Schließlich hat die Bestandsaufnahme Ansatzmöglichkeiten aufgezeigt, die eine differenzierte Analyse der langfristigen Wirkung der Kundenorientierung von Verkäufern ermöglichen. Die bisherigen Studien haben die daraus erwachsende Konfundierungsproblematik nicht hinreichend berücksichtigt. Insofern ist die Frage nach der langfristigen Wirkung des kundenorientierten Verkaufens auf den Verkaufserfolg und nach den dieser zugrunde liegenden differenzierten Wirkmechanismen unbeantwortet. Angesichts der praktischen Relevanz dieser Fragestellung verdient diese Thematik weitere Beachtung.

\section{Anhang}

Indikatoren zur Messung der hypothetischen Konstrukte ${ }^{\mathrm{a}, \mathrm{b}}$

\begin{tabular}{|c|c|}
\hline $\begin{array}{l}\text { Entscheidungsunterstützung (in Anlehnung an Saxe/Weitz 1982) } \\
\text { (Mittelwert }=4,6 ; \text { Standardabweichung }=1,66 \text { ) }\end{array}$ & FL \\
\hline Der Verkäufer versuchte, möglichst viel über meine Bedürfnisse zu erfahren. & 0,66 \\
\hline Der Verkäufer informierte mich umfassend über die Produkte. & 0,83 \\
\hline Der Verkäufer gab mir speziell solche Informationen, die mir die Produktauswahl erleichterten. & 0,74 \\
\hline Der Verkäufer bemühte sich sehr, mich bei meiner Kaufentscheidung zu unterstützen. & 0,67 \\
\hline $\begin{array}{l}\text { Aufbau positives Gesprächsklima (in Anlehnung an Donavan/Brown/Mowen 2004; } \\
\text { Lee/Dubinsky 2003) (Mittelwert }=4,7 \text {; Standardabweichung }=1,43 \text { ) }\end{array}$ & \\
\hline Der Verkäufer bemühte sich sehr um einen angenehmen Umgang mit mir. & 0,72 \\
\hline Der Verkäufer war sehr enthusiastisch. & 0,60 \\
\hline Der Verkäufer versuchte, auf der persönlichen Ebene eine Beziehung zu mir aufzubauen. & 0,63 \\
\hline Der Verkäufer gab mir zu erkennen, dass er mich schätzt. & 0,79 \\
\hline Der Verkäufer bemühte sich sehr, ein positives Gesprächsklima aufzubauen. & 0,79 \\
\hline $\begin{array}{l}\text { Vermeidung Abschlussdruck (in Anlehnung an Saxe/Weitz 1982) } \\
\text { (Mittelwert }=6,1 ; \text { Standardabweichung }=1,29 \text { ) }\end{array}$ & \\
\hline Der Verkäufer versuchte, mich von einem Kaufabschluss zu überzeugen. ${ }^{c}$ & 0,67 \\
\hline Durch das Vorgehen des Verkäufers empfand ich eine gewisse Art von Kaufdruck. ${ }^{c}$ & 0,89 \\
\hline Ich empfand den Verkäufer als aufdringlich. ${ }^{\mathrm{c}}$ & 0,85 \\
\hline Ich hatte den Eindruck, dass der Verkäufer mich zu einem sofortigen Kaufabschluss bringen wollte. ${ }^{\mathrm{c}}$ & 0,76 \\
\hline $\begin{array}{l}\text { Beratungszufriedenheit (in Anlehnung an Van Dolen et al. 2002) } \\
\text { (Mittelwert }=5,7 ; \text { Standardabweichung }=1,43 \text { ) }\end{array}$ & \\
\hline Alles in Allem, wie zufrieden waren Sie mit der Beratung durch den Berater? ${ }^{\mathrm{d}}$ & 0,84 \\
\hline Mit dem Ablauf des Beratungsgesprächs war ich sehr zufrieden. & 0,90 \\
\hline Ich fand die Art, wie der Verkäufer sein Beratungsgespräch geführt h & 0,93 \\
\hline
\end{tabular}

a: FL: Faktorladung

b: siebenstufige Skala (1=,,stimme gar nicht zu“; $7=$,stimme völlig zu“)

c: invers codiert

d: siebenstufige Skala (1=,,sehr unzufrieden“; 7=,,sehr zufrieden“) 


\section{Anmerkungen}

* Der Autor dankt den zwei anonymen Gutachtern für die wertvollen Anmerkungen und Anregungen zu einer früheren Version dieses Beitrags.

1 Aufgrund der unklaren Befundlage wird zum Zusammenhang zwischen Aufbau eines positiven Gesprächsklimas und Kaufentscheidung keine Hypothese formuliert. Gleichwohl wird ein möglicher direkter Zusammenhang im Rahmen der Empirie untersucht.

2 In diesem Sinne zeigten exploratorische Faktorenanalysen und Chow-Test (vgl. Chow 1960) weder für die Strukturen noch für die Wirkungen der Dimensionen signifikante Unterschiede in Abhängigkeit der Produktkategorien.

3 In diesem Sinne hatte sich im Rahmen einer qualitativen Vorstudie mit Kunden gezeigt, dass Kunden in jeder Betriebsform bereits sehr gute bis sehr schlechte Beratung erlebt hatten und insofern für eine Beratung ihr dafür präferiertes Geschäft aufsuchen. Entsprechende Auswertungen des vorliegenden Datensatzes deuteten erwartungsgemäß auf keinen Einfluss der Betriebsform auf die Ergebnisse hin.

4 Zur Prüfung von Common Method-Effekten bezüglich der Dimensionen des kundenorientierten Verkaufens wurde - wie in der Literatur vorgeschlagen (vgl. Podsakoff et al. 2003, S. 894) - in das Messmodell zusätzlich ein „Methodenfaktor“ integriert und das Modell neu geschätzt. Der Methodenfaktor wurde als Faktor erster Ordnung spezifiziert, ,,gemessen“ durch alle Indikatoren der drei Dimensionen. Dadurch erfasst dieser Faktor die systematische Varianz, die den itembezogenen Messungen über die drei Dimensionen hinweg gemein ist. Auch diese Analysen bestätigten die Güte des Messmodells.

5 Dass der Nachweis eines Zusammenhangs zwischen Entscheidungsunterstützung und Kaufentscheidung in Modell 2a knapp am 0,05-Signifikanznivau scheitert, ist dabei insofern unerheblich, als mediierende Effekte auch ohne nachweisbaren direkten Zusammenhang zwischen Prädiktor und Ergebnisvariable existieren können (vgl. Kenny/Kashy/Bolger 1998, S. 260).

6 Dieser gegenläufige Effekt führt dazu, dass der direkte Zusammenhang zwischen dem Aufbau eines positiven Gesprächsklimas und der Kaufentscheidung nicht signifikant ist (s. Tabelle 2, Modell 2a) (vgl. Kenny/ Kashy/Bolger 1998, S. 260, bes. FN 10).

7 Durch das Mean Centering der Variablen ist dieser Wert nicht skalierungsbedingt, sondern substantieller Natur, verursacht durch die nicht-symmetrische Verteilung der Variablen Vermeidung von Abschlussdruck (vgl. Cohen et al. 2003, S. 203). Diese ergibt sich daraus, dass die Verkäufer in der Mehrzahl der untersuchten Verkaufsinteraktionen - wenn überhaupt - einen eher geringen Verkaufsdruck als zweckmässig erachten. Dieser Befund steht insofern im Einklang mit der theoretischen Argumentation zum direkten Effekt.

8 Die dortigen Ergebnisse beziehen sich zwar explizit auf die moderierte Regression, lassen sich aber deshalb für die vorliegende Studie nutzen, weil ein polynomisches Regressionsmodell, wie das in der vorliegenden Studie, formal einen Spezialfall der moderierten Regression darstellt (vgl. Irwin/McClelland 2001, S. 101).

9 Zur Absicherung dieses Ergebnisses wurden auch Modelle gerechnet, in denen aus den Modellen 3a und 3b die Variablen Beratungszufriedenheit und Entscheidungsunterstützung entfernt wurden. Die Ergebnisse stützten in beiden Fällen einen Effekt des Quadratterms.

10 Diese Analysen wurden kausalanalytisch unter Einschluss des Methodenfaktors (s. Anmerkung 4) wiederholt. Die Ergebnisse zeigten keine substantielle Veränderung.

\section{Literatur}

Ajzen, I./Fishbein, M. (1973), Attitudinal and Normative Variables as Predictors of Specific Behavior, in: Journal of Personality \& Social Psychology, 27, 1, 41-57.

Albers, S. (1989), Kundennähe als Erfolgsfaktor, in: Albers, S./Hermann, H./Kahle, E./Kruschwitz, L./Perlitz, M. (Hrsg.), Elemente erfolgreicher Unternehmenspolitik in mittelständischen Unternehmen, Stuttgart, S. $101-122$.

Albers, S./Skiera, B. (1999), Regressionsanalyse, in: Herrmann, A./Homburg, Ch. (Hrsg.), Marktforschung, Wiesbaden, S. 203-236.

Baldauf, A./Cravens, D. W. (1999), Improving the Effectiveness of Field Sales Organizations: A European Perspective, in: Industrial Marketing Management, 28, S. 63-72.

Balderjahn, Ingo (1986), Das umweltbewußte Konsumentenverhalten, Berlin. 
Baron, R. M./Kenny, D. A. (1986), The Moderator - Mediator Variable Distinction in Social Psychological Research. Conceptual, Strategic and Statistical Considerations, in: Journal of Personality and Social Psychology, 51, S. 1173-1182.

Bateson, J. E. G. (1985), Perceived Control and the Service Encounter, in: Czepiel, J. A./Solomon, M. R./ Surprenant, C. F. (Eds.), The Service Encounter, Lexington, S. 67-82.

Bauer, R. A. (1967), Consumer Behavior as Risk Taking, in: Donald, F.C. (Ed.): Risk Taking and Information Handling in Consumer Behavior, Boston, S. 23-33 [Reprint aus: Hancock, R. S. (Ed.), Dynamic Marketing for a Changing World, Proceedings of the $43^{\text {rd }}$ Conference of the American Marketing Association, Boston 1960, S. 389-398.]

Bearden, W. O./Sharma, S./Teel, J. E. (1982), Sample Size Effects on Chi Square and Other Statistics Used in Evaluating Causal Models, in: Journal of Marketing Research, 19, S. 425-430.

Bettencourt, L. A./Brown, S. W./MacKenzie, S. B. (2005), Customer-oriented boundary-spanning behaviors: Test of a social exchange model of antecedents, in: Journal of Retailing, 81, S. 141-157.

Boles, J. S./Babin, B. J./Brashear, T. G./Brooks, C. (2001), An Examination of the Relationships between Retail Work Environments, Salesperson Selling Orientation-Customer Orientation and Job Performance, in: Journal of Marketing Theory and Practice, 9, 3, S. 1-13.

Boles, J. S./Johnson, J. T./Barksdale, Jr., H. C. (2000), How Salespeople Build Quality Relationships: A Replication and Extension, in: Journal of Business Research, 48, S. 75-81.

Bortz, J. (1999), Statistik für Sozialwissenschaftler, 5. Aufl., Berlin.

Brady, M. K./Cronin, Jr., J. J. (2001), Customer Orientation: Effects on Customer Service Perceptions and Outcome Behaviors, in: Journal of Service Research, 3, S. 241-251.

Brehm, J. W. (1966), A Theory of Psychological Reactance, New York.

Brehm, S. S./Brehm, J. W. (1981), Psychological Reactance, New York.

Brown, S. P. (1990), Use of Closed Influence Tactics by Salespeople: Incidence and Buyer Attributions, in: Journal of Personal Selling \& Sales Management, 10, Fall, S. 17-29.

Brown, T. J./Mowen, J. C./Donavan, D. T./Licata, J. W. (2002), The Customer Orientation of Service Workers: Personality Trait Effects on Self- and Supervisor Performance Ratings, in: Journal of Marketing Research, 39, February, S. 110-119.

Bruhn, M. (2003), Kundenorientierung, 2. Aufl., München.

Bunn, M. D. (1993), Taxonomy of Buying Decision Approaches, in: Journal of Marketing, 57, 1, S. 38-56.

Chow, G. C. (1960), Tests of Equality Between Sets of Coefficients in Two Linear Regressions, in: Econometrica, 28, S. 591-605.

Churchill, Jr., G. A./Suprenant, C. (1982), An Investigation into the Determinants of Customer Satisfaction, in: Journal of Marketing Research, 19, S. 491-504.

CIA (2006): The World Factbook, URL: http://www.cia.gov/cia/publications/factbook/fields/ 2177.html (Stand: 2.5.2006).

Cohen, J./Cohen, P./West, S. G./Aiken, L. S. (2003), Applied Multiple Regression/ Correlation Analysis for the Behavioral Sciences, $3^{\text {rd }}$ ed., Mahwah (NJ).

Crosby, L. A./Evans, K. R./Cowles, D. (1990), Relationship Quality in Services Selling: An Interpersonal Influence Perspective, in: Journal of Marketing, 54, July, S. 68-81.

DelVecchio, S./Zemanek, J./McIntyre, R./Claxton, R. (2004), Updating the Adaptive Selling Behaviours: Tactics to Keep and Tactics to Discard, in: Journal of Marketing Management, 20, S. 859-875.

DeVellis, Robert F. (1991), Scale Development.

Dixon, A. L. (1999), The Impact of Salesperson Behaviors on Performance: An Exploratory Study and an Empirical Examination, Diss., Ann Arbor.

Donavan, D. T./Brown, T. J./Mowen, J. C. (2004), Internal Benefits of Service-Worker Customer Orientation: Job Satisfaction, Commitment, and Organizational Citizenship Behaviors, in: Journal of Marketing, 68, January, S. 128-146.

Doney, P. M./Cannon, J. P. (1997), An examination of the nature of trust in buyer-seller relationships, in: Journal of Marketing, 61, 2, S. 35-51.

Evans, K. R./Kleine, R. E./Landry, T. D./Crosby, L. A. (2000), How First Impressions of a Customer Impact Effectiveness in an Initial Sales Encounter, in: Journal of the Academy of Marketing Science, 28, S. 512-526.

Flaherty, T. B. (1999), The Association Between Customer-Oriented Selling Performance with Outcome-Based and Judgmental-Based Measures of Salesperson Effectiveness, in: Noble, C. H. (Ed.), Developments in Marketing Science, Coral Gables, S. 221-225.

Fornell, C./Larcker, D. F. (1981), Evaluating Structural Equation Models with Unobservable Variables and Measurement Error, in: Journal of Marketing Research, 18, S. 39-50. 
Förster, E./Rönz, B. (1979), Methoden der Korrelations- und Regressionsanalyse, Berlin.

Franke, G. R./Park, J.-E. (2006), Salesperson Adaptive Selling Behavior and Customer Orientation: A MetaAnalysis, in: Journal of Marketing Research, 43, S. 693-702.

Gatignon, H./Vosgerau, J. (2006), Stochastic Moderated Regression: An Efficient Methodology for Estimating Parameters in Moderated Regression, Working Paper, Fontainebleau.

George, J. M. (1991), State or Trait: Effects of Positive Mood on Prosocial Behaviors at Work, in: Journal of Applied Psychology, 76, 2, S. 299-307.

Goff, B. G./Boles, J. S./Bellenger, D. N./Stojack, C. (1997), The Influence of Salespeople Selling Behaviors on Customer Satisfaction with Products, in: Journal of Retailing, 73, S. 171-183.

Grunert, K. G. (1978), Consumer Information Programs and the Concept of "Perceived Risk", in: Molt, W./ Hartmann, H. A./Stringer, P. (Eds.), Advances in Economic Psychology, Heidelberg, S. 161-174.

Haas, A. (2006a), Wie wirkt das kundenorientierte Verkaufen auf die Kundenzufriedenheit mit der Beratung? Eine Analyse unter Berücksichtigung der Interaktionen zwischen den Dimensionen des Verkäuferverhaltens, in: Marketing - Zeitschrift für Forschung und Praxis, 28, S. 231-241.

Haas, A. (2006b), Bestimmungsfaktoren des Beratungserfolges: Eine informationsökonomische Betrachtung und empirische Analyse im Handel, in: Zeitschrift für betriebswirtschaftliche Forschung, 58, S. 510536.

Haas, A. (2008), Kundenorientierung von Mitarbeitern: Forschungsstand und -perspektiven, in: Zeitschrift für Betriebswirtschaft, 78, 10, S. 1061-1100.

Hawes, J. M./Strong, J. T./Winick, B. S. (1996), Do Closing Techniques Diminish Prospect Trust?, in: Industrial Marketing Management, 25, S. 349-360.

Hennig-Thurau, T. (2004), Customer orientation of service employees: Its impact on customer satisfaction, commitment, and retention, in: International Journal of Service Industry Management, 15, S. 460-478.

Hisrich, R. D./Dornoff, R. J./Kernan, J. B. (1972), Perceived risk in store selection, in: Journal of Marketing Research, 9, S. 435-439.

Homburg, Ch. (1998), Kundennähe von Industriegüterunternehmen, 2. Aufl., Wiesbaden.

Homburg, Ch./Baumgartner, H. (1995), Beurteilung von Kausalmodellen. Bestandsaufnahme und Anwendungsempfehlungen, in: Marketing - ZFP, 17, S. 162-176.

Homburg, Ch./Bucerius, M. (2001), Kundenzufriedenheit als Managementherausforderung, in: Homburg, Ch. (Hrsg.), Kundenzufriedenheit, 4. Aufl., Wiesbaden, S. 51-83.

Homburg, Ch./Koschate, N./Hoyer,W. D. (2006), The Role of Cognition and Affect in the Formation of Customer Satisfaction: A Dynamic Perspective, in: Journal of Marketing, 70, July, S. 21-31.

Homburg, Ch./Pflesser, Ch. (1999), Konfirmatorische Faktorenanalyse, in: Herrmann, A./Homburg, Ch. (Hrsg.), Marktforschung, Wiesbaden, S. 413-437.

Homburg, Ch./Stock, R. (2001), Theoretische Perspektiven zur Kundenzufriedenheit, in: Homburg, Ch. (Hrsg.): Kundenzufriedenheit, 4. Aufl., Wiesbaden, S. 17-50.

Hosmer, D. W./Lemeshow, S. (2000), Applied Logistic Regression, $2^{\text {nd }}$ ed., New York.

Howe, V./Hoffman, K. D./Hardigree, D. W. (1994), The Relationship Between Ethical and Customer-Oriented Service Provider Behaviors, in: Journal of Business Ethics, 13, July, S. 497-506.

Irvin, J. R./McClelland, G. H. (2001), Misleading Heuristics and Moderated Multiple Regression Models, in: Journal of Marketing Ressearch, 38, S. 100-109.

Jaccard, J./King, G. W./Pomazal, R. (1977), Attitudes and Behavior: An Analysis of Specificity of Attitudinal Predictors, in: Human Relations, 30, S. 817-824.

Keillor, B. D./Parker, R. S./Pettijohn, C. E. (2000), Relationship-Oriented Characteristics and Individual Salesperson Performance, in: Journal of Business \& Industrial Marketing, 15, 1, S. 7-22.

Kempf, A./Ruenzi, S. (2006), Status Quo Bias and the Number of Alternatives: An Empirical Illustration from the Mutual Fund Industry, in: Journal of Behavioral Finance, 7, S. 204-213.

Kenny, D. A./Kashy, D. A./Bolger, N. (1998), Data Analysis in Social Psychology, in: Gilbert, D. T./Fiske, S. T./Lindzey, G. (Eds.), The Handbook of Social Psychology, Vol. I, $4^{\text {th }}$ ed., New York, S. 233-265.

Krafft, M. (1997), Der Ansatz der Logistischen Regression und seine Interpretation, in: Zeitschrift für Betriebswirtschaft, 67, 5/6, S. 625-642.

Krafft, M. (1999), Logistische Regression, in: Herrmann, A./Homburg, Ch. (Hrsg.), Marktforschung, Wiesbaden, S. 237-264.

Kraus, S. J. (1995), Attitudes and the Prediction of Behavior: A Meta-Analysis of the Empirical Literature, in: Personality and Social Psychology Bulletin, 21, S. 58-75.

Kroeber-Riel, W./Weinberg, P. (2003), Konsumentenverhalten, 8. Aufl., München.

Kutner, M. H./Nachtsheim, C. J./Neter, J. (2004), Applied Linear Regression Models, $4^{\text {th }}$ ed., Boston. 
Lee, S./Dubinsky, A. J. (2003), Influence of salesperson characteristics and customer emotion on retail dyadic relationships, in: International Review of Retail, Distribution \& Consumer Research, 13, 1, S. 21-36.

Licata, J. W./Mowen, J. C./Harris, E. G./Brown, T. J. (2003), On the Trait Antecedents and Outcomes of Service Worker Job Resourcefulness: A Hierarchical Model Approach, in: Journal of the Academy of Marketing Science, 31, 3, S. 256-271.

Limbeck, M. (2005), Zurück zum Verkaufen, in: acquisa, 10, S. 69.

McFarland, R. G./Challagalla, G. N./Shervani, T. A. (2006), Influence Tactics for Effective Adaptive Selling, in: Journal of Marketing, 70, October, S. 103-117.

Menon, K./Dubé, L. (2000), Ensuring Greater Satisfaction by Engineering Salesperson Response to Customer Emotions, in: Journal of Retailing, 76, S. 285-307.

Michaels, R. E./Day, R. L. (1985), Measuring Customer Orientation of Salespeople: A Replication With Industrial Buyers, in: Journal of Marketing Research, 22, S. 443-446.

Morrison, D. G. (1969), On the Interpretation of Discriminant Analysis, in: Journal of Marketing Research, 6, S. $156-163$.

Niebisch, P. (1993), Beobachtung und Beurteilung von Verkäuferverhalten, Salzburg.

Nunnally, J. C. (1978), Psychometric Theory, $2^{\text {nd }}$ ed., New York.

Obermiller, C./Spangenberg, E. R. (2000), On the Origin and Distinctness of Skepticism toward Advertising, in: Marketing Letters, 11, S. 311-322.

Olshavsky, R. W. (1973), Customer-Salesman Interaction in Appliance Retailing, in: Journal of Marketing Research, 10, S. 208-212.

Pham, M. T./Cohen, J. B./Pracejus, J. W./Hughes, G. D. (2001), Affect Monitoring and the Primacy of Feelings in Judgment, in: Journal of Consumer Research, 28, 2, S. 167-188.

Pieters, R./Bottschen, G./ Thelen, E. (1998), Customer Desire Expectations about Service Employees: An Analysis of Hierarchical Relations, in: Psychology \& Marketing, 15, S. 755-773.

Podsakoff, P. M./MacKenzie, S. B./Lee, J.-Y./Podsakoff, N. P. (2003), Common Method Biases in Behavioral Research: A Critical Review of the Literature and Recommended Remedies, in: Journal of Applied Psychology, 88, S. 879-903.

Reid, D. A./Pullins, E. B./Plank, R. E. (2002), The Impact of Purchase Situation on Salesperson Communication Behaviors in Business Markets, in: Industrial Marketing Management, 31, S. 205-213.

Roselius, T. (1971), Consumer Rankings of Risk Reduction Methods, in: Journal of Marketing, 35, January, S. $56-61$.

Samuelson, W./Zeckhauser, R. (1988), Status quo bias in decision making, in: Journal of Risk and Uncertainty, 1, S. 7-59.

Saxe, R./Weitz, B. A. (1982), The SOCO Scale: A Measure of the Customer Orientation of Salespeople, in: Journal of Marketing Research, 19, S. 343-351.

Schaefer, A. D./Pettijohn, C. E. (2006), The Relevance of Authenticity in Personal Selling: Is Genuineness an Asset or Liability?, in: Journal of Marketing Theory \& Practice, 14, 1, S. 25-35.

Schwarz, N. (1990), Feelings as Information: Informational and Motivational Functions of Affective States, in: Sorrentino, R./Higgins, E. T. (Eds.), Handbook of Motivation and Cognition: Foundations of Social Behavior, Vol. 2, New York, S. 527-561.

Schweitzer, M. (1994), Disentangling Status Quo and Omission Effects: An Experimental Analysis, in: Organizational Behavior and Human Decision Processes, 58, S. 457-476.

Schwepker, Jr., C. H. (2003), Customer-Oriented Selling: A Review, Extension, and Directions for Future Research, in: Journal of Personal Selling \& Sales Management, 23, S. 151-171.

Sheth, J. N. (1973), A Model of Industrial Buyer Behavior, in: Journal of Marketing, 37, S. 50-56.

Siguaw, J. A./Brown, G./Widing, II, R. E. (1994), The Influence of the Market Orientation of the Firm on Sales Force Behavior and Attitudes, in: Journal of Marketing Research, 31, February, S. 106-116.

Singley, R. B./Williams, M. R. (1995), Free Riding in Retail Stores: An Investigation of its Perceived Prevalence and Costs, in: Journal of Marketing Theory \& Practice, 3, 2, S. 64-74.

Stafford, J./Greer, T. V. (1965), Consumer Preferences for Types of Salesmen: A study of Independence-Dependence Characteristics, in: Journal of Relatives, 41, Summer, S. 27-33.

Statistisches Bundesamt (2006), Bevölkerung nach Geschlecht und Staatsangehörigkeit; URL: http://www. destatis.de/basis/d/bevoe/bevoetab4.php (Stand: 23.8.2006).

Stock, R. (2002), Kundenorientierung auf individueller Ebene: Das Einstellungs-Verhaltens-Modell, in: Die Betriebswirtschaft, 52, S. 59-76.

Stock, R. M./Hoyer, W. D. (2002), Leadership Style as Driver of Salespeoples' Customer Orientation, in: Journal of Market-Focused Management, 5, S. 355-376. 
Stock, R. M./Hoyer, W. D. (2005), An Attitude-Behavior Model of Salespeople's Customer Orientation, in: Journal of the Academy of Marketing Science, 33, S. 536-552.

Stock-Homburg, R. (2008), Die Rolle des marktorientierten Personalmanagements im Rahmen der Umsetzung marktorientierter Strategien: Eine empirische Untersuchung, in: Schmalenbachs Zeitschrift für betriebswirtschaftliche Forschung, 60, 2, S. 124-152.

Tversky, A./Shafir, E. (1992), Choice under conflict: The dynamics of deferred decision, in: Psychological Science, 3, S. 358-361.

Van Dolen, W./Lemmink, J./De Ruyter, K./De Jong, A. (2002), Customer - sales employee encounters: a dyadic perspective, in: Journal of Retailing, 78, S. 265-279.

Verhoef, P. C./Franses, P. H./Donkers, B. (2002), Changing Perceptions and Changing Behavior in Customer Relationships, in: Marketing Letters, 13, 2, S. 121-134.

Weiber, R./Adler, J. (1995), Positionierung von Kaufprozessen im informationsökonomischen Dreieck: Operationalisierung und verhaltenswissenschaftliche Prüfung, in: Zeitschrift für betriebswirtschaftliche Forschung, 47, S. 99-123. 


\section{Kann zu viel Kundenorientierung nachteilig sein? Eine Analyse der Wirkung der Kundenorientierung von Verkäufern auf die Kaufentscheidung}

\section{Zusammenfassung}

In diesem Beitrag wird analysiert, wie das kundenorientierte Verkäuferverhalten auf die Kaufentscheidung der Kunden wirkt. Die Analyse erfolgt auf der Ebene der Dimensionen des kundenorientierten Verkaufens, namentlich Entscheidungsunterstützung, Aufbau eines positiven Gesprächsklimas und Vermeidung von Abschlussdruck. Die Ergebnisse bestätigen die indirekte Wirkung des kundenorientierten Verkaufens auf die Kaufentscheidung über die Beratungszufriedenheit. Daneben können sowohl ein direkter umgekehrt u-förmiger Effekt der Vermeidung von Abschlussdruck auf die Kaufentscheidung als auch ein direkter negativer Einfluss des Aufbaus eines positiven Gesprächsklimas auf die Kaufentscheidung nachgewiesen werden. Zudem zeigt sich, dass der Aufbau eines positiven Gesprächsklimas den stärksten Einfluss auf die Beratungszufriedenheit ausübt.

\section{Can Too Much Customer Orientation Be Less Effective? An Analysis of the Effects of Customer-Oriented Selling on Customers' Decision to Buy}

\section{Summary}

This research investigates the effects of customer-oriented selling on customers' decision to buy. In so doing, the focus of the analysis is on the effects of the dimensions of customer-oriented selling, namely supporting customer decision-making, building a positive interaction climate, and avoiding pressure to close the sale. The findings of an empirical study support an indirect effect of customer-oriented selling on customers' decision to buy through customer satisfaction with the sales interaction. The results also support a direct inverted u-shaped effect of avoiding pressure to close the sale on customers' decision to buy as well as a direct negative effect of building a positive interaction climate on customers' decision to buy. And building a positive interaction climate was found to have the strongest effect on customer satisfaction with the sales interaction. 